\title{
Counting Transitive Relations with Two Ordered Pairs
}

\author{
Firdous Ahmad Mala \\ Department of Higher Education, Govt. Degree College Sopore, Baramulla, Jammu and Kashmir, India.
}

How to cite this paper: Firdous Ahmad Mala. (2021) Counting Transitive Relations with Two Ordered Pairs. Journal of Applied Mathematics and Computation, 5(4), 247-251.

DOI: $10.26855 /$ jamc.2021.12.002

Received: September 6, 2021

Accepted: September 30, 2021

Published: October 13, 2021

*Corresponding author: Firdous Ahmad Mala, Department of Higher Education, Govt. Degree College Sopore, Baramulla, Jammu and Kashmir, India.

Email: firdousmala@gmail.com

\begin{abstract}
Enumerating transitive relations on a set seems very difficult. There is no known formula that counts the number of transitive relations on a set with $n$ elements. However, OEIS, which is an online repository of more than 350,000 integer sequences, provides the number of transitive relations for all sets with fewer than nineteen elements. The problem is also closely related to some other open problems in Combinatorics including the enumeration of partial orders, quasiorders and topologies on a finite set. In this paper, we visualize the distribution of transitive relations with two ordered as a matrix. Consequently, an explicit biquadratic formula is obtained for the number of transitive relations with two ordered pairs. It is also shown that for sufficiently large sets, relations with two ordered pairs that are not transitive are extremely rare. The technique provided herein could conveniently be generalized to the enumeration of transitive relations with three or more ordered pairs.
\end{abstract}

\section{Keywords}

Transitive relations, Enumeration

\section{Introduction}

Let $S$ be a non-empty set. Any subset of $S \times S$ is called a relation on $S$. A relation on $S$ is said to be transitive if and only if $\forall x, y, z \in S,(x, y),(y, z) \in S \Rightarrow(x, z) \in S$.

Regarding the number of transitive relations on a set with n elements, Pfeiffer attempted counting them using automorphism groups [1]. Recently, in F. A. Mala [2], several interesting recursive relations and lower bounds for the number of transitive relations on a set were obtained. Despite such attempts, there is no known formula that explicitly counts the number of transitive relations on a set with $\mathrm{n}$ elements. OEIS [3] enlists the number of transitive relations on sets with less than 19 elements. Interestingly, several other open enumeration problems are very closely related to the problem of enumerating transitive relations on a set. Efforts have been made to make progress in these directions, and some very useful information is available in M. Y. Kizmaz [4].

\section{Main Discussion}

Definition 1: Let $[n]$ denote the set of first $n$ natural numbers. $\forall i, j \in[n]$, let $A_{i j}$ denote the set of transitive relations with two ordered pairs on $[n]$, one of the ordered pairs being $(i, j)$.

Definition 2: For $n \in \mathbb{N}$, consider the square matrix $T_{n}$ given by 


$$
T_{n}=\left(t_{i j}\right)_{n} \text {, where } t_{i j}=\left|A_{i j}-\underset{\substack{m \leq i, n \leq y \\(m, n) \neq(i, j)}}{U} A_{m n}\right|
$$

This means that while calculating $t_{i j}$ in $T_{n}$, we first calculate $t_{a b}$ and then $t_{c d}$ if either $a<c$ or $a=c, b<d$ taking due care that no transitive relation with two ordered pairs is counted twice. In other words, we are warding-off double counts.

For example,

$$
\begin{aligned}
& \mathrm{T}_{2}=\left(\begin{array}{ll}
\mathrm{t}_{11} & \mathrm{t}_{12} \\
\mathrm{t}_{21} & \mathrm{t}_{22}
\end{array}\right)=\left(\begin{array}{ll}
3 & 1 \\
1 & 0
\end{array}\right) \\
& \mathrm{T}_{3}=\left(\begin{array}{lll}
\mathrm{t}_{11} & \mathrm{t}_{12} & \mathrm{t}_{13} \\
\mathrm{t}_{21} & \mathrm{t}_{22} & \mathrm{t}_{23} \\
\mathrm{t}_{31} & \mathrm{t}_{32} & \mathrm{t}_{33}
\end{array}\right)=\left(\begin{array}{lll}
8 & 4 & 3 \\
4 & 4 & 1 \\
2 & 1 & 0
\end{array}\right) \\
& \mathrm{T}_{4}=\left(\begin{array}{cccc}
\mathrm{t}_{11} & \mathrm{t}_{12} & \mathrm{t}_{13} & \mathrm{t}_{14} \\
\mathrm{t}_{21} & \mathrm{t}_{22} & \mathrm{t}_{23} & \mathrm{t}_{24} \\
\mathrm{t}_{31} & \mathrm{t}_{32} & \mathrm{t}_{33} & \mathrm{t}_{34} \\
\mathrm{t}_{41} & \mathrm{t}_{42} & \mathrm{t}_{43} & \mathrm{t}_{44}
\end{array}\right)=\left(\begin{array}{cccc}
15 & 9 & 8 & 7 \\
9 & 10 & 5 & 4 \\
6 & 5 & 5 & 1 \\
3 & 2 & 1 & 0
\end{array}\right) \\
& \mathrm{T}_{5}=\left(\begin{array}{ccccc}
\mathrm{t}_{11} & \mathrm{t}_{12} & \mathrm{t}_{13} & \mathrm{t}_{14} & \mathrm{t}_{15} \\
\mathrm{t}_{21} & \mathrm{t}_{22} & \mathrm{t}_{23} & \mathrm{t}_{24} & \mathrm{t}_{25} \\
\mathrm{t}_{31} & \mathrm{t}_{32} & \mathrm{t}_{33} & \mathrm{t}_{34} & \mathrm{t}_{35} \\
\mathrm{t}_{41} & \mathrm{t}_{42} & \mathrm{t}_{43} & \mathrm{t}_{44} & \mathrm{t}_{45} \\
\mathrm{t}_{51} & \mathrm{t}_{52} & \mathrm{t}_{53} & \mathrm{t}_{54} & \mathrm{t}_{55}
\end{array}\right)=\left(\begin{array}{ccccc}
24 & 16 & 15 & 14 & 13 \\
16 & 18 & 11 & 10 & 9 \\
12 & 11 & 12 & 6 & 5 \\
8 & 7 & 6 & 6 & 1 \\
4 & 3 & 2 & 1 & 0
\end{array}\right)
\end{aligned}
$$

Since while calculating the entries of matrix $T_{n}$, double counts were taken due care of, it is evident that the number of transitive relations with two ordered pairs is $\sum_{\mathrm{i}, \mathrm{j}=1}^{\mathrm{n}} \mathrm{t}_{\mathrm{ij}}$. Thus, the number of transitive relations with two ordered pairs on a two-element set, being simply the sum of the entries of $\mathrm{T}_{2}$, is 5 . Similarly, the number of transitive relations with two ordered pairs on a three-element set is 27 . We go on to show that the number of transitive relations with two ordered pairs is given by a biquadratic polynomial in $\mathrm{n}$.

\section{Preliminary Results}

Theorem 1: The sum of the diagonal entries of $T_{n}$ is given by the cubic

$$
\sum_{i=1}^{n} t_{i i}=\frac{n\left(n^{2}-1\right)}{2}
$$

Theorem 2: The lower triangular sum of $T_{n}$ is given by the biquadratic

$$
\sum_{\substack{i, j=1 \\ i>j}}^{n} t_{i j}=\frac{n(n-1)\left(n^{2}-n+1\right)}{6}
$$

Theorem 3: The upper triangular sum of $T_{n}$ is given by the biquadratic

$$
\sum_{\substack{i, j=1 \\ i<j}}^{n} t_{i j}=\frac{n(n-1)\left(2 n^{2}-5 n-5\right)}{6}
$$

\section{Main Theorem}

Theorem 4: The number of transitive relations with two ordered pairs on a set with $n$ elements is

$$
\frac{n^{4}-2 n^{3}+4 n^{2}-3 n}{2}=\frac{n(n-1)\left(n^{2}-n+3\right)}{2}
$$

Since the empty relation is transitive and so is each relation with only one ordered pair, the number of transitive relations with at-most two ordered pairs on a set with $n$ elements is $n^{2}+1$ more than the number of transitive relations with two ordered pairs. Consequently, we have the following. 
Corollary 1: The number of transitive relations with at most two ordered pairs on a set with $n$ elements is

$$
\frac{n^{4}-2 n^{3}+4 n^{2}-3 n}{2}+n^{2}+1=\frac{n^{4}-2 n^{3}+8 n^{2}-3 n+4}{2}
$$

\section{Proofs of the theorems}

\section{Proof of theorem 1}

We write down $T_{n}$ by filling up its diagonal entries. The ordered pair $(1,1)$ coupled with any other ordered pair gives rise to a transitive relation with two ordered pairs. The same is true for all the ordered pairs $(i, i), 1 \leq i \leq n$. Thus, $t_{11}=n^{2}-1=(n-1)(n+1), t_{22}=(n-2)(n+1), t_{33}=(n-3)(n+1), \cdots, t_{n n}=(n-n)(n+1)=0$. This helps complete the diagonal entries of $T_{n}$.

$$
T_{n}=\left(\begin{array}{ccccc}
n^{2}-1 & t_{12} & t_{13} & \cdots & t_{1 n} \\
t_{21} & (n-2)(n-1) & t_{23} & \cdots & t_{2 n} \\
t_{31} & t_{32} & (n-3)(n-1) & \cdots & t_{3 n} \\
\vdots & \vdots & \vdots & \vdots & \vdots \\
t_{n 1} & t_{n 2} & t_{n 3} & \cdots & 0
\end{array}\right)
$$

Consequently, the sum of the diagonal entries of $T_{n}$ is

$$
\begin{aligned}
& \sum_{i=1}^{n} t_{i i}=(n-1)(n+1)+(n-2)(n+1)+(n-3)(n+1)+\cdots+(n-n)(n+1) \\
& =(n+1)[(n-1)+(n-2)+(n-3)+\cdots+0]=(n+1) \sum_{0}^{n-1} r=\frac{(n+1)(n-1)}{2}=\frac{n^{2}-1}{2}
\end{aligned}
$$

\section{Proof of theorem 2}

We now start writing down the lower triangular entries in $T_{n}$, that is, we calculate $t_{i j}$ for every $i>j$. We observe that the first entry in the bottom row is $n-1$ and each subsequent entry is 1 less.

$$
T_{n}=\left(\begin{array}{ccccc}
t_{11} & t_{12} & t_{13} & \cdots & t_{1 n} \\
t_{21} & t_{22} & t_{23} & \cdots & t_{2 n} \\
t_{31} & t_{32} & t_{33} & \cdots & t_{3 n} \\
\vdots & \vdots & \vdots & \vdots & \vdots \\
n-1 & n-2 & n-3 & \cdots & 0
\end{array}\right)
$$

This is simply because each ordered pair $(n, i), 1 \leq i \leq n-1$ coupled with any one of the subsequent ordered pairs gives rise to a transitive relation with two ordered pairs. Interestingly, for each entry in the lower triangular region that is exactly above the entry $(n, i), 1 \leq i \leq n-2$, we observe that $t_{i j}=t_{i+1 j}+(n-1)$. The lower triangular region in $T_{3}$ and $T_{4}$, for example, are respectively

$$
T_{3}=\left(\begin{array}{ccc}
t_{11} & t_{12} & t_{13} \\
4 & t_{22} & t_{23} \\
2 & 1 & t_{33}
\end{array}\right) \text { and } T_{4}=\left(\begin{array}{cccc}
t_{11} & t_{12} & t_{13} & t_{14} \\
9 & t_{22} & t_{23} & t_{24} \\
6 & 5 & t_{33} & t_{34} \\
3 & 2 & 1 & t_{44}
\end{array}\right) .
$$

With this observation in mind, we complete the lower triangle of $T_{n}$ as follows:

$$
T_{n}=\left(\begin{array}{cccc}
t_{11} & t_{12} & \cdots & t_{1 n} \\
(n-1)(n-1) & t_{22} & \cdots & t_{2 n} \\
\vdots & \vdots & \vdots & \vdots \\
2(n-1) & (n-2)+(n-1) & \cdots & \vdots \\
n-1 & n-2 & \cdots & 0
\end{array}\right)
$$

Consequently, the lower triangular sum of $T_{n}$ is 


$$
\begin{aligned}
& \sum_{\substack{i, j=1 \\
i>j}}^{n} t_{i j}=(n-1)^{2}+(n-2)^{2}+\cdots+0+(n-1)[1+(1+2)+\cdots+(1+2+3+\cdots+(n-2)] \\
& =\frac{n(n-1)(2 n-1)}{6}+(n-1)\left(\sum_{r=1}^{n-2}(1+2+3+\cdots+r)\right) \\
& =\frac{n(n-1)(2 n-1)}{6}+(n-1)\left(\sum_{r=1}^{n-2} \frac{r(r+1)}{2}\right)=\frac{n(n-1)(2 n-1)}{6}+\frac{n-1}{2}\left(\sum_{r=1}^{n-2}\left(r^{2}+r\right)\right) \\
& =\frac{n(n-1)(2 n-1)}{6}+\frac{n-1}{2}\left(\sum_{r=1}^{n-2} r^{2}+\sum_{r=1}^{n-2} r\right) \\
& =\frac{n(n-1)(2 n-1)}{6}+\frac{n-1}{2}\left(\frac{(n-2)(n-1)(2 n-3)}{6}+\frac{(n-2)(n-1)}{2}\right)=\frac{n(n-1)\left(n^{2}-n+1\right)}{6}
\end{aligned}
$$

\section{Proof of theorem 3}

We now start writing down the lower triangular entries in $T_{n}$, that is, we calculate $t_{i j}$ for every $i<j$. We observe that the second entry in the top row is $(n-1)^{2}$ and each subsequent entry is 1 less.

$$
T_{n}=\left(\begin{array}{ccccc}
t_{11} & (n-1)^{2} & (n-1)^{2}-1 & \cdots & (n-1)^{2}-(n-2) \\
t_{21} & t_{22} & t_{23} & \cdots & t_{2 n} \\
t_{31} & t_{32} & t_{33} & \cdots & t_{3 n} \\
\vdots & \vdots & \vdots & \vdots & \vdots \\
n-1 & n-2 & n-3 & \cdots & 0
\end{array}\right)
$$

Interestingly, for sum of the entries of the first row exceeds the sum of the entries of the first column by $(n-2)(1+2+\cdots+(n-2))$. The sum of the entries of the second row exceeds the sum of the entries of the second column by $(n-2)(1+2+(n-3))$ and so on.

Consequently,

$$
\begin{gathered}
\sum_{\substack{i, j=1 \\
i<j}}^{n} t_{i j}=\sum_{\substack{i, j=1 \\
i>j}}^{n} t_{i j}+(n-2)\left(\sum_{r=1}^{n-2}(1+2+3+\cdots r)\right)=\frac{n(n-1)\left(n^{2}-n+1\right)}{6}+(n-2)\left(\sum_{r=1}^{n-2} \frac{r(r+1)}{2}\right) \\
=\frac{n(n-1)\left(n^{2}-n+1\right)}{6}+\frac{(n-2)}{2}\left(\sum_{r=1}^{n-2}\left(r^{2}+r\right)\right) \\
=\frac{n(n-1)\left(n^{2}-n+1\right)}{6}+\frac{(n-2)}{2}\left(\sum_{r=1}^{n-2} r^{2}+\sum_{r=1}^{n-2} r\right) \\
=\frac{n(n-1)\left(n^{2}-n+1\right)}{6}+\frac{n-2}{2}\left(\frac{(n-2)(n-1)(2 n-3)}{6}+\frac{(n-2)(n-1)}{2}\right) \\
=\frac{n(n-1)\left(n^{2}-n+1\right)}{6}+\frac{(n-2)^{2}(n-1) n}{12}=\frac{n(n-1)}{6}\left(n^{2}-n+1+n^{2}-4 n+4\right)=\frac{n(n-1)\left(2 n^{2}-5 n+5\right)}{6}
\end{gathered}
$$

\section{Proof of theorem 4}

Counting the required number of transitive relations with two ordered pairs is now fairly simple. All we need to do is add the diagonal sum, the lower triangular sum and the upper triangular sum of $T_{n}$.

Using theorems 1, 2 and 3, the required sum is

$$
\sum_{i, j=1}^{n} t_{i j}=\sum_{i=1}^{n} t_{i i}+\sum_{\substack{i, j=1 \\ i>j}}^{n} t_{i j}+\sum_{\substack{i, j=1 \\ i<j}}^{n} t_{i j}
$$




$$
\begin{aligned}
& =\frac{n\left(n^{2}-1\right)}{2}+\frac{n(n-1)\left(n^{2}-n+1\right)}{6}+\frac{n(n-1)\left(2 n^{2}-5 n+5\right)}{6} \\
& =\frac{n(n-1)}{6}\left(3(n+1)+n^{2}-n+1+2 n^{2}-5 n+5\right)=\frac{n(n-1)}{6}\left(3 n^{2}-3 n+9\right)=\frac{n(n-1)\left(n^{2}-n+3\right)}{2}
\end{aligned}
$$

Corollary 2: For the matrix $T_{n}=\left[t_{i j}\right]_{n}, j=i+1 \Rightarrow t_{i j}=t_{j i}$.

Proof: The corollary is immediate from theorem 3.

Corollary 3: If $t_{2}(n)$ is the number of transitive relations on an $n$-set with two ordered pairs, and $r_{n}(n)$ is the total number of relations on an $n$-set with two ordered pairs, then $\lim _{n \rightarrow \infty} \frac{t_{2}(n)}{r_{2}(n)}=1$.

Proof: Clearly, $r_{2}(n)=\left(\begin{array}{c}n^{2} \\ 2\end{array}\right)=\frac{n^{2}\left(n^{2}-1\right)}{2}$ and $t_{2}(n)=\frac{n(n-1)\left(n^{2}-n+3\right)}{2}$.

$$
\text { Thus, } \lim _{n \rightarrow \infty} \frac{t_{2}(n)}{r_{2}(n)}=\lim _{n \rightarrow \infty} \frac{n(n-1)\left(n^{2}-n+3\right)}{n^{2}\left(n^{2}-1\right)}=\lim _{n \rightarrow \infty} \frac{n^{2}-n+3}{n^{2}+n}=1 .
$$

\section{Conclusion}

In this paper, a technique to enumerate transitive relations on a set with a specific number of ordered pairs is proposed. The technique is demonstrated on the case $n=2$. It is proved that the number of transitive relations with two ordered pairs on an $n$-set is a biquadratic polynomial in $n$.

\section{References}

[1] Pfeiffer, Götz. (2004). Counting transitive relations. Journal of Integer Sequences, (2004) https://cs.uwaterloo.ca/ journals/JIS/VOL7/Pfeiffer/pfeiffer6.html.

[2] Mala, F. A. (2021). On the number of transitive relations on a set. Indian J Pure Appl Math, (2021). https://doi.org/10.1007/s13226-021-00100-0.

[3] OEIS, Sloane, Neil J. A. and The OEIS Foundation Inc. The on-line encyclopedia of integer sequences, A006905. https://oeis.org/A006905.

[4] Kizmaz, M. Y. (2019). On the number of topologies on a finite set. Algebra and Discrete Mathematics, 27(1), 50-57. http://mi.mathnet.ru/adm691. 